\title{
Utility of multi-detector row computed tomography angiography versus Doppler in localization of perforators of anterolateral thigh flaps
}

\author{
Chandan N. Jadhav ${ }^{1}$, Surinder Singh Makkar ${ }^{1}$, Gautam Biswas ${ }^{1}$, Niranjan Khandelwal ${ }^{2}$ \\ ${ }^{1}$ Department of Plastic Surgery, Postgraduate Institute of Medical Education and Research, Chandigarh 160012, India. \\ ${ }^{2}$ Department of Radio Diagnosis, Postgraduate Institute of Medical Education and Research, Chandigarh 160012, India.
}

Address for correspondence: Dr. Chandan N. Jadhav, 4/126, Terrace Road, Perth, Western Australia 6000, Australia. E-mail: chandansurgery@gmail.com

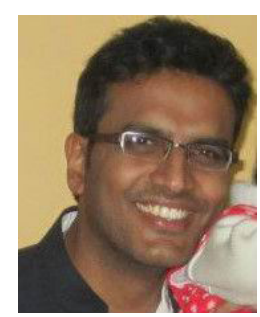

Dr. Chandan N. Jadhav, MBBS, MS, MCh, Plastic Reconstructive and Aesthetic Surgeon, Fellowship Hand and Microsurgery (Australia), DAFPRS Aesthetic Surgery Fellowship (Switzerland), Member of Editorial Board for Micromedicine-ABMS and reviewer for EJPS, Burns \& trauma. Have several publications in indexed peer reviewed journals. Current research interests are in ADSC in fat grafts.

\section{ABSTRACT}

Aim: Anterolateral thigh (ALT) flap is widely used in reconstruction of various defects. Preoperative imaging facilitates perforator mapping, overcoming intraoperative uncertainty. The purpose of this study was to investigate the utility of multi-detector row computed tomography angiography (MDCTA) and a handheld Doppler in locating ALT perforators. Methods: Twenty patients were randomized into two groups. Group 1 patients received MDCTA and Doppler studies whereas Group 2 received only a Doppler study. The number, location, course, and source of all cutaneous and sizable perforators were compared with intraoperative findings. Surgeons' stress levels during flap harvest and flap harvest time were compared. Results: MDCTA findings correlated well with intraoperative findings for perforator type and segmental distribution with $100 \%$ concordance. Doppler alone had a $52 \%$ rate of concordance. The sensitivity and specificity for MDCTA in demonstrating the presence of perforators were $85.71 \%$ and $97.22 \%$, respectively; whereas for Doppler alone the sensitivity and specificity were $80 \%$ and $87.91 \%$, respectively. In demonstrating perforator source, MDCTA showed a sensitivity of $100 \%$ and specificity of $91.66 \%$, with $100 \%$ accuracy. Sensitivity and specificity for sizable perforators were $90 \%$ each, with $88.88 \%$ accuracy. Doppler studies were unable to provide this information. Comparison of surgeon stress levels showed no differences between the two groups, although the time for flap harvest was significantly shorter in Group 1. Conclusion: MDCTA compared to Doppler is more sensitive, specific, and accurate with respect to location, course, and source of perforators.

Key words:

Anterolateral thigh; multi-detector row computed tomography angiography; perforator

\begin{tabular}{|l|l|}
\hline \multicolumn{2}{|c|}{ Access this article online } \\
\hline Quick Response Code: & Website: \\
\hline & www.parjournal.net \\
\cline { 2 - 3 } & \\
\hline
\end{tabular}

This is an open access article distributed under the terms of the Creative Commons Attribution-NonCommercial-ShareAlike 3.0 License, which allows others to remix, tweak and build upon the work non-commercially, as long as the author is credited and the new creations are licensed under the identical terms.

For reprints contact: service@oaepublish.com

How to cite this article: Jadhav CN, Makkar SS, Biswas G, Khandelwal N. Utility of multi-detector row computed tomography angiography versus Doppler in localization of perforators of anterolateral thigh flaps. Plast Aesthet Res 2016;3:52-8.

Received: 31-05-2015; Accepted: 19-11-2015 


\section{INTRODUCTION}

The anterolateral thigh (ALT) flap has become an increasingly popular reconstructive option due to its versatility of design, ability to be thinned and minimal donor site morbidity. The major limitation of this flap is the uncertainty in predicting perforator anatomy due to variability in perforator size and course. ${ }^{[1]}$ Formal analysis of these variations has not been adequately explored. Many authors have described the common location of ALT perforators as a tool in guiding flap harvest, but few have highlighted the inconsistencies. ${ }^{[1]}$ To improve operative planning, preoperative imaging is being increasingly utilized. In the past, Doppler ultrasound has been used for perforator mapping, with most studies demonstrating high sensitivity but poor accuracy and high interobserver variability. Despite improvements in ultrasound technology, this technique has been frequently abandoned, and there are trends toward performing no preoperative localization at all. ${ }^{[2]}$ Multi-detector row computed tomography angiography (MDCTA) has become a powerful noninvasive alternative to conventional digital subtraction angiography in preoperative imaging. ${ }^{[3-5]}$ The utility of MDCTA for preoperative planning in comparison with Doppler and effectiveness of the $\mathrm{ABC}$ system in preoperative perforator localization has not been studied in an adequate number of patients in the Indian population. The present randomized controlled study was been designed to investigate the utility of preoperative imaging in the localization of perforators and design of the skin paddle. Flap harvest time, surgeon's stress levels, and operative outcome were also assessed.

\section{METHODS}

\section{Patients}

In patients undergoing free ALT flaps, the goals were (1) to compare the number, location, course, and source of cutaneous perforators with the use of preoperative MDCTA and a handheld Doppler device, with intraoperative observation as the gold standard; and (2) to compare the subjective stress levels of the surgeon during perforator dissection and flap harvest time in patients who had preoperative MDCTA versus those who did not.

The pilot study done between January and December 2011 included all patients who required a free ALT flap. Patients with a documented history of significant atherosclerotic disease with blockage at the level of the infrarenal aorta, lower limb infections, scars, prior surgery to the thighs, and preexisting renal disease, diabetes, or cardiovascular disease were excluded.

\section{Handheld Doppler localization}

All patients underwent preoperative perforator localization using a handheld audible Doppler probe (Huntleigh Healthcare, $8 \mathrm{MHz}$, Cardiff, UK) performed by an independent assessor who was blinded to the MDCTA findings. The patient was placed in the supine position with the leg straight in a neutral position. A line was drawn connecting the anterior superior iliac spine (ASIS) to the superolateral corner of the patella (hereafter referred to as the AP line). The distance between these two points were measured, and the AP line was divided into 10 equal parts (hereafter referred to as segments) for the purpose of standardization between individuals and comparison. The Doppler signals were assessed at three main sites with a radius of $3 \mathrm{~cm}$. A signal at the midpoint of the AP line corresponded to segment 5 , while the others $5 \mathrm{~cm}$ proximal and distal to midpoint corresponded to segments 4 and 6 , respectively. The most audible signals were marked each time by the same observer in all patients. The distance of the Doppler signals from the AP line were plotted on the $\mathrm{X}$-axis (horizontal) and from a perpendicular drawn at the midpoint of the AP line, on the Y-axis.

\section{Randomization into two groups}

Following Doppler assessment, patients were randomized into two groups using computer-generated random numbers. Blocks of four were used to aid adequacy in randomization. In the first group (Group 1), preoperative mapping of location, number, source vessel, and course of all perforators of the ALT using an MDCTA was performed. In the second group (Group 2), no preoperative MDCTA was performed.

\section{MDCTA}

MDCTA was performed using a 64-detector row computed tomography scanner with the following parameters: 120 $\mathrm{kVp}$, 80-120 mA, gantry rotation time $0.4 \mathrm{~s}$, detector configuration $16 \mathrm{~mm} \times 1 \mathrm{~mm}, 23 \mathrm{~mm}$ table travel per rotation, $512 \times 512$ matrix, and 180-240 field of view. All scans were performed with intravenous (IV) administration of $100 \mathrm{~mL}$ of nonionic iodinated contrast medium with a concentration of $300 \mathrm{mg} / \mathrm{mL}$ and injected at a rate of $4 \mathrm{~mL} / \mathrm{s}$ through an 18-gauge IV catheter inserted into an antecubital vein. A bolus tracking technique was employed to obtain images from the point of bifurcation of the abdominal aorta to the level of the knee joint. The volumetric data acquired was then retrospectively used to reconstruct images with a slice thickness of $2 \mathrm{~mm}$ and a reconstruction interval of $0.75 \mathrm{~mm}$ in a soft tissue kernel. Ten radio-opaque markers ( $1 \mathrm{~cm}$ diameter plastic buttons) were placed at equal intervals along the AP line to depict each segment that assisted in accurate localization of perforators on preoperative MDCTA, which were plotted on the X-axis and Y-axis + or - symbols were used to depict the distances as plotted on the graph keeping the midpoint of intersection of AP line as $(0,0)$. These were then compared to the intraoperative findings.

\section{Operative technique}

All patients underwent harvest of a free ALT flap using the anterior approach as described by Song et al. ${ }^{[6]}$ and Koshima et al. ${ }^{[7]}$ Seven out of 10 patients in Group 1 and 9 out of 10 patients in Group 2 underwent subfascial dissection while suprafascial dissection was performed in the remainder of cases. During flap harvest, the location of each cutaneous perforator was marked with a needle at a specified distance from the perforator through the fascia 
into the skin. A mark was then made on the skin paddle at this site. This point was then plotted on the $\mathrm{X}$ - and $\mathrm{Y}$-axis after resuturing the skin paddle (subtracting the specified distance) to eliminate the obliquity of perforator entrance secondary to flap retraction/sagging. Care was taken to identify all perforators to the skin paddle which were preserved until the very end before committing to base the flap on the sizable perforators.

\section{Surgeons' stress level}

Surgeon's perceived (subjective) stress level during flap harvest was scored on a four-point visual analog scale (VAS) and recorded as follows:

- Grade 1 = no stress (preoperative perforator location matched intraoperative findings with only minor discrepancies $(<2 \mathrm{~cm})$ in perforator location);

- Grade 2 = mild stress (discrepancy measured more than $2 \mathrm{~cm}$ in perforator location between preoperative and intraoperative findings);

- Grade 3 = moderate stress (gross difference in the perforator location, source, and course); and

- Grade 4 = severe stress (no perforator was present, or inadvertent perforator injury occurred during dissection).

Time taken for flap harvest and surgical outcome were also noted.

\section{Statistical analysis}

Statistical Package for the Social Science, version 19, IBM (2010) was used. The Kolmogorov-Smirnov test was applied to determine the distribution of data, and if data was skewed, Mann-Whitney test was applied. For comparison of categorical data, the Fischer exact and Chi-squared tests were applied. Kappa inter-rater agreement was applied to determine agreement between the preoperative findings of MDCTA versus Doppler using intraoperative findings as the gold standard.

\section{RESULTS}

A total of 20 patients over a period of 1 year who underwent free ALT flap coverage at our hospital were allocated randomly into two groups.

\section{Patient demographics}

In Group 1, the mean age of patients was 37.5 years \pm 11.49 years, and in Group 2, it was 43 years \pm 14.29 years $(P=0.35)$. There was a total of six patients with post head and neck cancer resection defects ( 3 in each group) while one patient in the Group 1 had invasive aspergillosis of the maxillary sinus. Eight patients had lower limb traumatic defects (5 in Group 1 and 3 in Group 2), and five patients had upper limb traumatic defects ( 1 in Group 1 and 4 in Group 2). Traumatic limb defects accounted for $65 \%$ of cases while nontraumatic defects accounted for $35 \%$.

\section{Anterolateral thigh flap characteristics}

Four cutaneous ALT flaps (3 in Group 1 and 1 in Group 2), ten fasciocutaneous flaps ( 4 in Group 1 and 6 in Group 2), five musculocutaneous (MC) flap ( 2 in Group 1 and 3 in Group 2), and one vastus lateralis muscle flap (in Group 1) were performed. Skin paddle size varied between $63 \mathrm{~cm}^{2}$ and $264 \mathrm{~cm}^{2}$ in Group 1 and between $90 \mathrm{~cm}^{2}$ and $220 \mathrm{~cm}^{2}$ in Group 2 with a mean of $173.78 \mathrm{~cm}^{2}$ and $170.10 \mathrm{~cm}^{2}$, respectively $(P=0.89)$.

\section{Perforator number and type}

MDCTA picked up all seven septocutaneous (SC) perforators, 4/7 MC perforators of which $1 / 4$ were semiseptocutaneous (SSC). There were no differences between MDCTA and intraoperative findings for the distribution of type of perforators $(P=0.68)$.

\section{Perforator source}

Perforators were compared based on their source vessel: descending branch of the lateral circumflex femoral artery (DBLCFA), anteromedial thigh (AMT) perforator arising from the DBLCFA, the transverse branch of the lateral circumflex femoral artery (TBLCFA), or the oblique branch of the lateral circumflex femoral artery (OBLCFA). MDCTA accurately detected 8 out of 9 perforators arising from the DBLCFA and 3 of the 4 perforators arising from the TBLCFA. Two AMT perforators were identified intraoperatively (both in Group 2). There were no differences between preoperative MDCTA and intraoperative findings for the source vessel and origin of the perforators $(P=0.832)$.

\section{Sizable perforators}

In our study, any perforator over $0.8 \mathrm{~mm}$ was considered to be sizable. ${ }^{[8]}$ MDCTA detected all sizable SC perforators, $4 / 5$ sizable MC perforators of which $1 / 2$ was SSC. Doppler signals localized sizable perforators accurately in only 2 of 9 patients in Group 1 and 4 of 11 patients in Group 2. Sizable perforators were further compared based on their source vessel, i.e. DBLCFA, DBLCFA-AMT, TBLCFA, or OBLCFA. MDCTA localized all sizable perforators arising from the DBLCFA and TBLCFA. Overall sensitivity and specificity of MDCTA in demonstrating the sizable perforator in segments 4 and 5 was $90 \%$ and had an accuracy of $88.88 \%$ with a kappa value of 0.78 (good agreement) for each segment.

\section{Concordance of MDCTA versus Doppler for perforator localization}

A difference of more than $2 \mathrm{~cm}$ between preoperative localization and intraoperative findings was considered to be discordant. In Group 1, MDCTA had a concordance level of $100 \%(12 / 12)$ while Doppler had concordance of $46 \%(6 / 13)$. Overall concordance of Doppler was only $52 \%(13 / 25)$. This further establishes the accuracy of MDCTA in localization of perforators. The BlandAltman plot [Figure 1] was used to depict the inter-rater agreement between the two variables (MDCTA with intraoperative findings in the first plot and Doppler with intraoperative findings in the second plot) by plotting the average of the distance of perforators noted by both the variables against its difference from the mean. This demonstrates that the values were 
closer to the mean in the MDCTA-intraoperative plot, indicating a good agreement in the locations of the perforators as compared to the Doppler-intraoperative plot which was dispersed away from the mean.

\section{Surgeons' stress levels}

A VAS was used to record the level of stress experienced by the surgeon during flap harvest. The difference

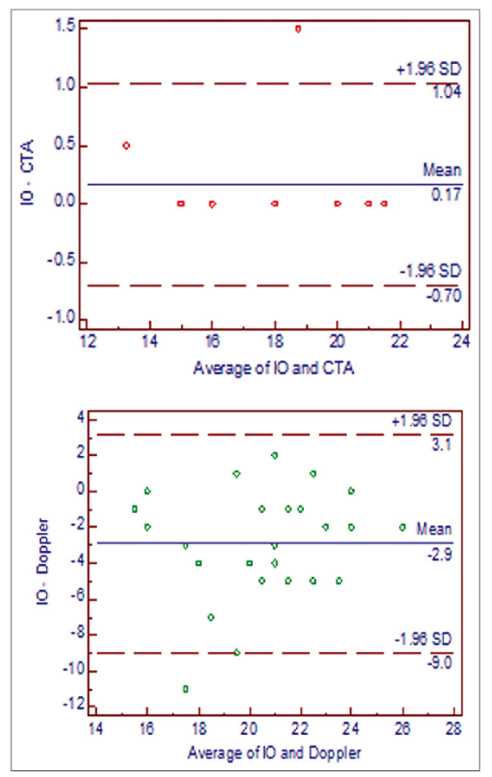

Figure 1: Bland-Altman plot for determining agreement between multi-detector row computed tomography angiography, Doppler, and intraoperative perforator location. IO: intraoperative; SD: standard deviation between the mean VAS of Group 1 (2.1) and Group 2 (2.5) was not statistically significant $(P=0.63)$. The difference between mean flap harvest time of Group 1 (87.5 min) and Group 2 (117.5 min) was not statistically significant $(P=0.28)$. However, operator bias cannot be ruled out. Surgeon A (chief surgeon) performed an equal number of surgeries (five) in each group, of which two cases in each group required an intramuscular perforator dissection for perforators arising from the DLBCFA. The difference between mean flap harvest time for Surgeon A in Group 1

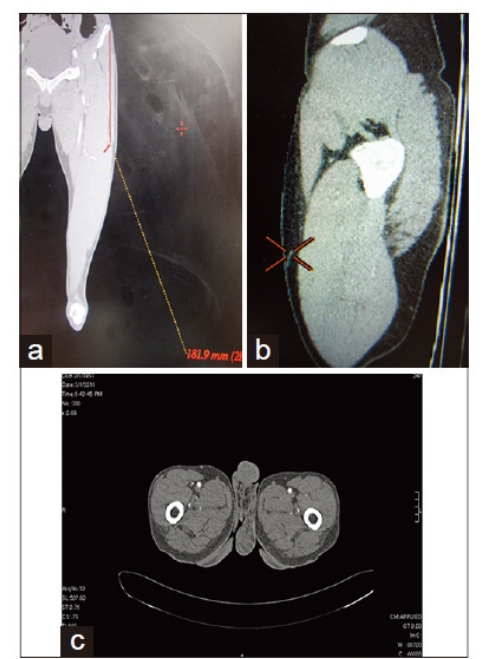

Figure 2: (a-c) Case 9: MDCTA coronal, sagittal, and axial section showing TBLCFAP-s $18 \mathrm{~cm}$ from ASIS. MDCTA: multi-detector row computed tomography angiography; ASIS: anterior superior iliac spine; TBLCFAP-s: septocutaneous perforator from transverse branch of lateral circumflex femoral artery through spectrum

Table 1: Perioperative details

\begin{tabular}{|c|c|c|c|c|c|c|c|c|c|}
\hline \multirow[t]{2}{*}{ Patien } & \multirow[t]{2}{*}{ Diagnosis } & \multirow[t]{2}{*}{ Group } & \multicolumn{2}{|c|}{ Perforator } & \multicolumn{2}{|c|}{ Concordance } & \multirow{2}{*}{$\begin{array}{l}\text { Sizable } \\
\text { Perforator }\end{array}$} & \multirow[t]{2}{*}{ VAS } & \multirow{2}{*}{$\begin{array}{l}\text { Time } \\
\text { (min) }\end{array}$} \\
\hline & & & Type CTA & Type 10 & Doppler & MDCTA & & & \\
\hline $\begin{array}{l}1 \\
2\end{array}$ & $\begin{array}{l}\text { Grade 3B fracture lower one-third leg } \\
\text { Carcinoma buccal mucosa }\end{array}$ & $\begin{array}{l}1 \\
1\end{array}$ & $\begin{array}{l}\text { No perforator } \\
M C\end{array}$ & $\begin{array}{l}\text { No perforator } \\
\text { MC, SC, SC }\end{array}$ & $\begin{array}{l}\text { No } \\
\text { Yes, yes }\end{array}$ & $\begin{array}{l}\text { Yes } \\
\text { Yes }\end{array}$ & $\begin{array}{l}\text { VL muscle } \\
\text { TBLCFAP-vl }\end{array}$ & $\begin{array}{l}4 \\
3\end{array}$ & $\begin{array}{l}115 \\
80\end{array}$ \\
\hline 3 & Carcinoma buccal mucosa & 1 & SC & $\mathrm{SC}, \mathrm{SC}$ & Yes, yes & Yes & DBLCFAP-S & 1 & 60 \\
\hline 4 & $\begin{array}{l}\text { Type 3A maxillectomy defect } \\
\text { (invasive aspergillosis) }\end{array}$ & 1 & SC, SC & SC & No, no & Yes, yes & DBLCFAP-s & 3 & 100 \\
\hline 5 & Carcinoma buccal mucosa & 1 & MC & MC & Yes & Yes & DBLCFAP-vl & 1 & 75 \\
\hline 6 & Traumatic sole defect & 1 & SC & SSC & Yes & Yes & OBLCFAP-vl & 2 & 115 \\
\hline 7 & Forearm electrical burns & 1 & $\mathrm{MC}, \mathrm{SC}$ & MC, SSC & No & Yes & DBLCFAP-vl & 2 & 100 \\
\hline 8 & Heel unstable scar & 1 & SC & SC & No & Yes & TBLCFAP-s & 2 & 55 \\
\hline 9 & Traumatic heel defect & 1 & SC & SC & No & Yes & TBLCFAP-s & 2 & 110 \\
\hline 10 & Traumatic heel defect & 1 & & SSC & No & Yes & DBLCFAP-vl & 1 & 65 \\
\hline 11 & Carcinoma buccal mucosa & 2 & NA & MC & No & NA & DBLCFAP-vl & 1 & 85 \\
\hline 12 & Traumatic elbow defect & 2 & NA & SSC, SC & No, yes & NA & TBLCFAP-vl & 4 & 95 \\
\hline 13 & Grade $3 B$ fracture lower $1 / 3$ leg & 2 & NA & $\mathrm{MC}, \mathrm{MC}$ & No & NA & DBLCFAP-S & 3 & 85 \\
\hline 14 & $\begin{array}{l}\text { Type } 4 \text { maxillectomy defect } \\
\text { (carcinoma maxillary sinus) }\end{array}$ & 2 & NA & $\mathrm{MC}$ & Yes & NA & $\begin{array}{l}\text { DBLCFAP-vl+ } \\
\text { AMTP }\end{array}$ & 4 & 125 \\
\hline 15 & Grade 3B fracture lower $1 / 3$ leg & 2 & NA & $\mathrm{MC}, \mathrm{SC}$ & No & NA & DBLCFAP-vl & 1 & 105 \\
\hline 16 & Hand degloving & 2 & NA & $\mathrm{MC}, \mathrm{SC}(\mathrm{AMT})$ & Yes & NA & DBLCFAP-S & 4 & 180 \\
\hline 17 & Open wrist joint and hand defect & 2 & NA & $\mathrm{SC}$ & Yes, no & NA & TBLCFAP-vl & 4 & 215 \\
\hline 18 & Grade $3 \mathrm{~B}$ fracture mid $1 / 3 \mathrm{leg}$ & 2 & NA & SC & Yes & NA & DBLCFAP-S & 2 & 95 \\
\hline 19 & Carcinoma buccal mucosa & 2 & NA & SC & Yes & NA & DBLCFAP-S & 1 & 85 \\
\hline 20 & Forearm contour correction & 2 & NA & SC & Yes & NA & DBLCFAP-S & 1 & 105 \\
\hline
\end{tabular}

AMTP: anteromedial thigh perforator; MDCTA: multi-detector row computed tomography angiography; DBLCFAP-s: descending branch lateral circumflex femoral artery perforator through septum; DBLCFAP-vl: descending branch lateral circumflex femoral artery perforator through vastus lateralis; IO: intraoperative; MC: musculocutaneous; NA: not applicable; OBLCFAP-vl: oblique branch lateral circumflex femoral artery perforator through vastus lateralis; SC: septocutaneous; SSC: semi-septocutaneous; TBLCFAP-vl: transverse branch lateral circumflex femoral artery perforator through vastus lateralis; TBLCFAP-s: transverse branch lateral circumflex femoral artery perforator through septum; VAS: visual analog scale; VL: vastus lateralis; AMT: anteromedial thigh 


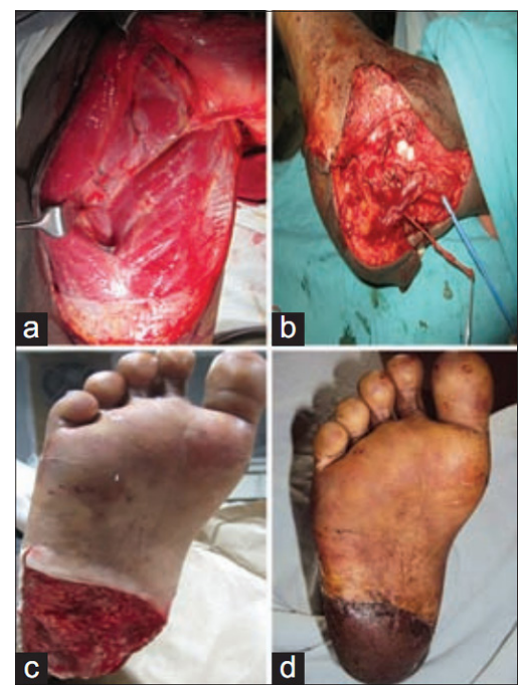

Figure 3: Case 9: (a) Sensate ALT flap, LCFN included; (b) intraoperative sizable septocutaneous perforator (TBLCFAP-s) was $18 \mathrm{~cm}$ from ASIS as determined preoperatively by MDCTA; (c) 8 weeks postoperative showing well settled sensate ALT flap; (d) posttraumatic heel defect with exposed calcaneus. ALT: anterolateral thigh; LCFN: lateral cutaneous femoral nerve; ASIS: anterior superior iliac spine; MDCTA: multi-detector row computed tomography angiography; TBLCFAP-s: septocutaneous perforator from transverse branch of lateral circumflex femoral artery through spectrum

(71 min) and Group 2 (95 min) was statistically significant $(P=0.046)$. Perioperative details are shown in Table 1. Figures 2 and 3 are representative of case 9, and Figure 4 is representative of case 4 .

\section{DISCUSSION}

The vascular basis of the ALT flap has been extensively studied since its introduction by Song et al. ${ }^{[6]} 30$ years ago. Although anatomy of the lateral circumflex femoral source vessel is quite consistent, the perforators to the skin territory can have multiple variations. ${ }^{[9]}$ Various imaging modalities have been used to predict the course and location of the perforators, of which MDCTA has been found to be the most consistent..$^{[9]}$

\section{Perforator(s) number and type}

In the current study, MDCTA did not affect the choice of limb (whether right or left side) for flap harvest as compared to a study by Rozen et al. ${ }^{[1]}$ The current study demonstrated an average of 1.45 perforators per limb, with $51.75 \%$ (15/29) SC and 48.25\% (14/29) MC perforators. There were no perforators in $5 \%$ (one) of the patients. These findings differed from those published by Kimata et al., ${ }^{[10]}$ in which $81.9 \%$ of the perforators were MC, $18.9 \%$ were SC, and there was no perforator in $5 \%$ of the patients. In the current study, SSC comprised $37.1 \%(5 / 14)$ of the total number of MC perforators, which differs from the study of Kim et al. ${ }^{[9]}$ which showed SSC in $4.6 \%$ of patients. However, MDCTA failed to accurately label the SSC perforator in 2 patients and instead identified them as SC. This is consistent with the observation that more careful evaluation is required for identification of SSC perforators. ${ }^{[9]}$

\section{Perforator source}

There was no difference between MDCTA and intraoperative findings for the source of perforators, indicating the efficacy of MDCTA. One patient had a
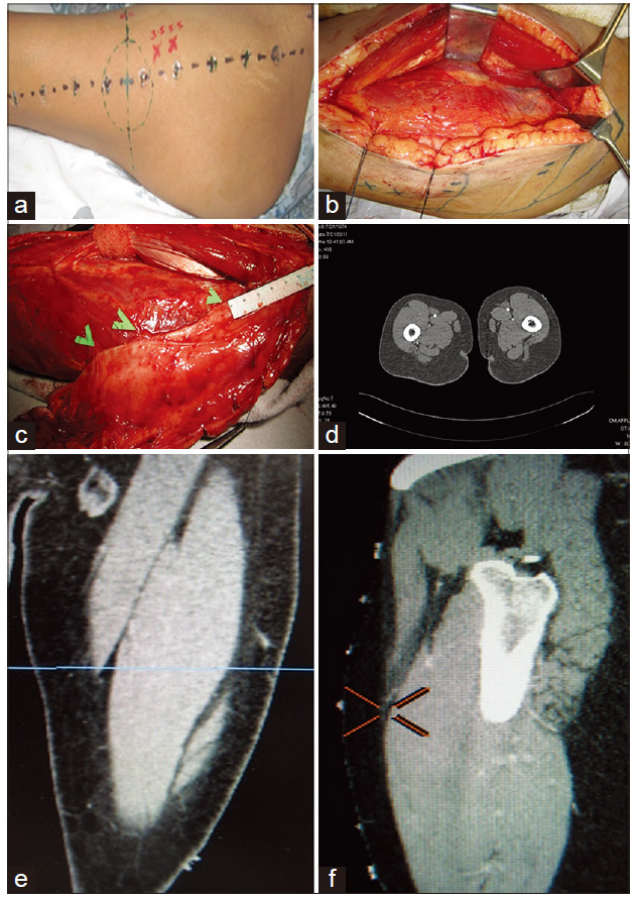

Figure 4: Case 4: (a) "X" denotes Doppler signal, "•" denotes MDCTA preoperative perforator localization; (b) plastic buttons at segments of thigh along AP line. Doppler signal at $3.5 \mathrm{~cm}$ and $5.5 \mathrm{~cm}$ above midpoint of AP line; (c) MDCTA axial section showing TBLCFAP; (d) sizable semi-septocutaneous perforator (TBLCFAP-sSc) $15 \mathrm{~cm}$ from ASIS and two other septocutaneous perforators DBLCFAP-s which correlated with preoperative MDCTA; (e) and (f) same TBLCFAP seen in coronal and sagittal sections. MDCTA: multi-detector row computed tomography angiography; TBLCFAP-ssc: semi-septocutaneous perforator arising from transverse branch of lateral circumflex femoral artery; DBLCFAP-s: septocutaneous perforator arising from descending branch of lateral circumflex femoral artery

perforator arising from an OBLCFA (a branch of DBLCFA), which on MDCTA was thought to be an intramuscular perforator arising from the DBLCFA. This was similar to the study by Wong et al., ${ }^{[1]}$ in which the oblique branch predominantly arises from the DBLCFA.

\section{Segmental distribution of perforators}

Most of the perforators in this study were concentrated in segments 4 and $5(24 / 29)$ [Table 2], which differed from the study by Kim et al.,$^{|9|}$ in which perforators were concentrated in segments 5 and 6 . There was no difference in the segmental distribution of perforators when comparison was made between MDCTA and intraoperative findings. However, the difference was statistically significant when compared to findings with Doppler localization $(P=0.034)$ [Table 2]. When the intraoperative segmental distributions of perforator types were analyzed, it was noted that both SC $(n=8)$ and MC $(n=7)$ perforators were concentrated in segment 5 . However, SSC perforators were localized in proximal segments $3(n=2)$ and segment $4(n=2)$. This differed from the study by Lin et al., ${ }^{[12]}$ in which the SC perforators were located in more proximal segments than the MC perforators. When the perforator quadrant was mapped in consistent with similar study by Yu et al. ${ }^{[2]}$ MDCTA was independent of body mass index (BMI).

Sensitivity, specificity, accuracy of MDCTA in segments 4 and 5 on the right and left thighs, most of perforators were 
found in the upper outer quadrant of the thigh, as opposed to the study of Kim et al., ${ }^{[9]}$ which showed perforators primarily in the lower outer quadrant of the thigh.

\section{Sensitivity, specificity, accuracy of Doppler versus MDCTA in localization}

Yu et al., ${ }^{[2]}$ in 2006, evaluated the accuracy of handheld Doppler and found it to be overly sensitive, poorly specific, and inaccurate in locating perforators. The current study showed an overall sensitivity of $74 \%$ and specificity of $80.86 \%$ for Doppler in the demonstration of the presence of a perforator. Lin et al. ${ }^{[12]}$ conducted another study in 2011 in which MDCTA demonstrated a sensitivity of $74 \%$ and specificity of $90 \%$ in 16 patients. The current study showed a sensitivity and specificity for MDCTA of $85.71 \%$ and $97.22 \%$ in demonstrating the presence of a perforator [Table 3], while that for Doppler alone was $80 \%$ and $87.91 \%$, respectively in Group 1 [Table 4]. MDCTA was superior to Doppler in accurate localization of the perforators in segments 3, 4, and 6. However, no difference was seen in segment 5 . Doppler showed less accuracy in localizing perforators when the BMI increased.

\section{Perforator source}

The sensitivity of MDCTA in the detection of the source of the perforators decreased from proximal to distal while the specificity remained high in all segments, similar to a study by Garvey et al. ${ }^{[13]}$ Perforators appeared to be better visualized when surrounded by subcutaneous fat, which decreases from proximal to distal in the thigh. MDCTA had $100 \%$ sensitivity, $91.66 \%$ specificity, and $100 \%$ accuracy in demonstrating the source of perforators in segments 4 and 5 arising from the TBLCFA and in segment 5 for perforators arising from the DBLCFA. Our study showed an overall kappa value of 1 (very good agreement) for MDCTA in demonstrating the source of perforators, similar to the study by Garvey et al., ${ }^{\mid 13]}$ The Doppler was unable to provide any information regarding source vessel, course, and size of perforators.

\section{Surgeons' stress levels}

This study attempted to objectively classify the stress level of a surgeon during flap harvest and perforator dissection. Taylor et al.$^{[3,14]}$ used the retrograde VAS to assess the surgeons' stress levels during deep inferior epigastric artery perforator (DIEP) dissection. This was subjectable to recall bias and hence unreliable. In our study, the surgeon's operative stress score was analyzed prospectively. The difference in mean scores of the surgeon's stress level during flap harvest was not statistically significant, likely secondary to the small number of cases. However, the mean flap harvest time for Surgeon A was significantly less in Group $1(P=0.046)$. No similar studies have been reported in literature.

\section{Complications}

This study found no significant differences in flap survival or donor site complications in patients who underwent MDCTA versus those who did not $(P=$ 0.26). This is in contrast to other studies of the DIEP flap, where preoperative MDCTA significantly decreased the incidence of marginal necrosis and donor site morbidity. ${ }^{[14]}$ However, it would probably be incorrect to extrapolate data obtained from an abdominal donor site and compare it to a thigh donor site. In the current study,

Table 2: Segmental distribution of perforators

\begin{tabular}{|c|c|c|c|c|c|c|c|c|}
\hline \multirow{3}{*}{$\begin{array}{l}\text { Perforator } \\
\text { segment }\end{array}$} & \multicolumn{4}{|c|}{ Intraoperative } & \multicolumn{2}{|c|}{ Preoperative } & \multicolumn{2}{|c|}{$P$} \\
\hline & \multicolumn{2}{|c|}{ Group 1} & \multicolumn{2}{|c|}{ Group 2} & \multirow{2}{*}{$\frac{\text { MDCTA }}{n}$} & \multirow{2}{*}{$\begin{array}{c}\text { Doppler } \\
n\end{array}$} & \multirow[t]{2}{*}{ MDCTA } & \multirow[t]{2}{*}{ Doppler } \\
\hline & $n$ & Percentage & $n$ & Percentage & & & & \\
\hline 3 & 1 & 7.14 & 2 & 13.33 & 1 & 0 & & \\
\hline 4 & 5 & 35.71 & 4 & 26.66 & 6 & 2 & & \\
\hline 5 & 7 & 50.00 & 8 & 53.33 & 4 & 22 & 0.666 & 0.034 \\
\hline 6 & 1 & 7.14 & 1 & 6.33 & 0 & 4 & & \\
\hline 7 & 0 & 0.00 & 0 & 0.00 & 0 & 1 & & \\
\hline
\end{tabular}

MDCTA: multi-detector row computed tomography angiography

Table 3: Sensitivity, specificity, and accuracy of MDCTA to demonstrate the presence of a perforator in Group 1

\begin{tabular}{|c|c|c|c|c|c|c|}
\hline Segment & Sensitivity \% & $95 \% \mathrm{Cl}$ & Specificity \% & $95 \% \mathrm{Cl}$ & Accuracy $\%$ & K \\
\hline 3 & 100 & $5.46-100$ & 100 & $71.65-100$ & 100 & NA \\
\hline 4 & 100 & $46.29-100$ & 88.89 & $50.67-99.4$ & 92.8 & 0.85 \\
\hline 5 & 57.14 & $20.23-88.19$ & 100 & $56.09-100$ & 78.5 & 0.78 \\
\hline 6 & NA & NA & 100 & $71.66-100$ & 92.8 & NA \\
\hline Overall & 85.71 & & 97.22 & & & \\
\hline
\end{tabular}

$\mathrm{Cl}$ : confidence interval; NA: not applicable; MDCTA: multi-detector row computed tomography angiography

Table 4: Sensitivity, specificity, and accuracy of handheld Doppler to demonstrate the presence of a perforator in Group 1

\begin{tabular}{|c|c|c|c|c|c|c|}
\hline Segment & Sensitivity \% & $95 \% \mathrm{Cl}$ & Specificity \% & $95 \% \mathrm{Cl}$ & Accuracy & K \\
\hline 4 & 40 & $7.26-82.96$ & 100 & $62.88-100$ & 78.87 & 0.46 \\
\hline 5 & 100 & $56.01-100$ & 71.43 & $30.25-94.89$ & 85.71 & 0.71 \\
\hline 6 & 100 & $54.6-100$ & 92.31 & $62.08-99.60$ & 92.85 & 0.63 \\
\hline Overall & 80 & & 87.91 & & & \\
\hline
\end{tabular}

$\mathrm{Cl}$ : confidence interval 
2 flaps in Group 2 underwent complete necrosis. Minor complications including marginal flap necrosis (2 cases) and infection (one case) were noted in Group 1 and were managed conservatively.

\section{Prudent observations and surgical outcomes of this study}

- The largest skin paddle harvested measured $264 \mathrm{~cm}^{2}$ and survived. However, two flaps underwent complete necrosis (Group 2). In one, the MC perforator was injured, while in the other patient, the AMT perforator was dominant, but eccentric to the skin paddle designed which was based on a false localization by the Doppler signal. There was no perforator from the DBLCFA and the skin paddle had to be shifted proximally to include the TBLCFA perforator (TBLCFAP), which in turn had a tortuous intramuscular course and was inadvertently injured. Preoperative MDCTA could have picked up this anomaly, allowing the flap to be based on the AMT perforator. A thoraco-umbilical flap was performed as a salvage flap in this case. Two flaps had marginal necrosis (Group 1), one of which occurred secondary to a problem with the anastomosis. The recipient vessel posterior tibial artery had three episodes of vasospasm despite revision of the anastomosis. This was attributed to the subacute phase of injury. One patient in Group 2 with a defect of the upper and middle third of the leg had his anastomosis performed in the subacute phase of injury and distal to the zone of injury without any complications

- One patient in Group 1, who was diabetic, had delayed total flap loss after 2 weeks due to a necrotizing soft-tissue infection. This is a very rare complication which has not yet been reported

- In one patient in Group 2, the skin paddle was shifted $6 \mathrm{~cm}$ proximally as a sizable perforator was noted arising from the TBLCFA, which was more proximal than the proposed signal given by Doppler

- Three patients in Group 1 underwent intramuscular perforator dissection as opposed to only one patient in Group 2, as MDCTA provided a roadmap of the course and source vessel of a sizable perforator

- In one patient who required a sensate ALT flap for reconstruction of a heel defect, the skin paddle was planned based on the TBLCFAP through the septum, which was sizable SC perforator as shown on MDCTA, though there was also a sizable MC perforat or (DBLCFA perforator-vastus lateralis) which was found more distally. The skin paddle was therefore designed proximally to include the lateral cutaneous femoral nerve of the thigh. MDCTA in this case significantly influenced preoperative planning

- MDCTA of one patient did not show any sizable perforators in one thigh, which was consistent with intraoperative finding. However, the Doppler gave a good audible signal in the same thigh, and an ALT flap was planned. When no perforator was identified, vastus lateralis muscle only flap was done. This was the index case in Group 1

- In one patient with a Type 3A maxillectomy defect following invasive aspergillosis, a flap with two skin paddles was initially planned, one for the palate and cheek based on the DBLCFA through septum and the other for the nasal lining based on the TBLCFAP through septum as noted on MDCTA.
Hence, MDCTA was useful in planning the reconstruction of complex defects requiring multiple paddles, similar to the study done by Garvey et al. ${ }^{[13]}$

In conclusion, preoperative MDCTA as compared to Doppler was more sensitive, specific, and accurate with respect to the location, course, and source vessel of all perforators. This study demonstrates that preoperative MDCTA provides us with all the information required to make a choice regarding design of the skin paddle and also reduces the flap harvest time which was statistically significant. Our study showed that preoperative MDCTA lowered the surgeons' stress level during perforator dissection. Further studies with large number of patients are required to reach statistically significant conclusions. The trends shown toward the benefits of performing preoperative MDCTA are nonetheless encouraging.

\section{Financial support and sponsorship}

Nil.

\section{Conflicts of interest}

There are no conflicts of interest.

\section{REFERENCES}

I. Rozen WM, Ashton MW, Pan WR, Kiil BJ, McClure VK, Grinsell D, Stella DL, Corlett RJ. Anatomical variations in the harvest of anterolateral thigh flap perforators: a cadaveric and clinical study. Microsurgery 2009;29:16-23.

2. Yu P,Youssef A. Efficacy of the handheld Doppler in preoperative identification of the cutaneous perforators in the anterolateral thigh flap. Plastic Reconstr Surg 2006; I 18:928-33.

3. Taylor GI,Doyle M,McCarten G.The Doppler probe for planning flaps:anatomical study and clinical applications. Br J Plast Surg 1990;43: I-16.

4. Rosson GD,Williams CG, Fishman EK, Singh NK.3D CT angiography of abdominal wall vascular perforators to plan DIEAP flaps. Microsurgery 2007;27:64I-6.

5. Hamdi M,Van LK,Van HE.Advances in autogenous breast reconstruction: the role of preoperative perforator mapping. Ann Plast Surg 2007;58:18-26.

6. Song YG, Chen GZ, Song YL.The free thigh flap: a new free flap concept based on the septocutaneous artery. Br J Plast Surg 1984;37:149-59.

7. Koshima I, Fukuda H,Utunomiya R, Soeda S.The anterolateral thigh flap; variations in its vascular pedicle. BrJ Plast Surg 1989;42:260-2.

8. Koshima I, Nanba Y, Tsutsui T, Takahashi Y, Itoh S, Fujitsu M. Minimal invasive lymphaticovenular anastomosis under local anesthesia for leg lymphedema: is it effective for stage III and IV? Ann Plast Surg 2004;53:26I-6.

9. Kim EK, Kang BS, Honget JP.The distribution of the perforators in the anterolateral thigh and the utility of multidetector row computed tomography angiography in preoperative planning. Ann Plast Surg 2010;65:155-60.

10. Kimata Y, Uchiyama K, Ebihara S, Nakatsuka T, Harii K. Anatomic variations and technical problems of the anterolateral thigh $\mathrm{fl}$ ap: a report of 74 cases. Plast Reconstr Surg 1998;102:1517-23

II. Wong CH, Wei FC, Fu B, Chen YA, Lin JY. Alternative vascular pedicle of the anterolateral thigh flap: the oblique branch of the lateral circumflex femora artery. Plast Reconstr Surg 2009; 123:57I-7.

12. Lin SJ, Rabie A, Yu P. Designing the anterolateral thigh flap without preoperative Doppler or imaging.J Reconstr Microsurg 2010;26:67-72.

13. Garvey PB, Selber JC, Madewell JE, Bidaut L, Feng L, Yu P. A prospective study of preoperative computed tomographic angiography for head and neck reconstruction with anterolateral thigh flaps. Plast Reconstr Surg 201 I;127:150514.

14. Rozen WM, Anavekar NS, Ashton MW, Stella DL, Grinsell D, Bloom RJ, Taylor $\mathrm{Gl}$. Does the preoperative imaging of perforators with CT angiography improve operative outcomes in breast reconstruction? Microsurgery 2008;28:516-23. 